\title{
Bức ảnh "mờ mịt": mỹ cảm khoa học, đức hy sinh và vận hội của một tạp chí
}

\author{
Hồ Mạnh Toàn \\ A.I. for Social Data Lab, SSHPA System
}

Hà Nội, 16-04-2019

Ngày 10 tháng 4 vừa qua, khi cả thế giới chiêm ngưỡng bức ảnh đầu tiên chụp trực tiếp hố đen vũ trụ lịch sử, thì Heather Schewedel của Slate lại đánh giá bức ảnh quá xấu, và các nhà khoa học cần chỉnh sửa nó cho đẹp hơn [1]. Đây là một nhận định chủ quan, và cũng "có vẻ hợp lý" nếu chỉ nhìn từ góc độ hình ảnh sản phẩm cuối cùng được sản xuất ra. Ngay cả những nhà nghiên cứu góp sức mang lại bức ảnh cũng phải nhận xét nó khá mờ mịt, và không có tính thẩm mỹ cao. Tuy nhiên, sự kỳ vĩ của công trình này, nếu được tìm hiểu kỹ càng, sẽ mang lại những đánh giá hoàn toàn khác. Đầu tư cho khoa học đã mang lại những giá trị phi thường, không đơn giản là đo đếm thông thường [2].

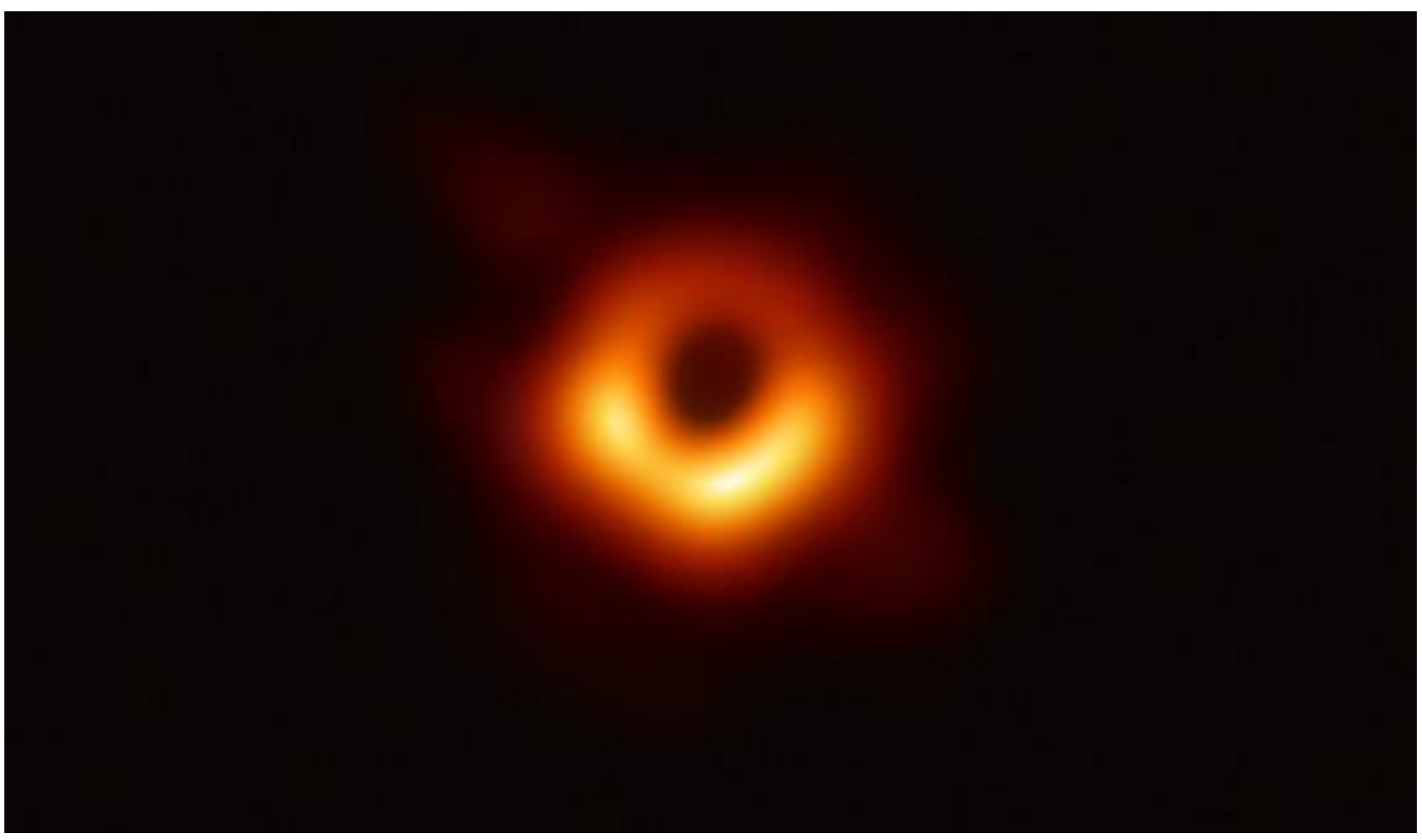

Bản nét với độ phân giải cao có thể được download tại Event Horizon Telescope (Nguồn: Event Horizon Telescope)

Không ai có thể được phép quên mất rằng, bức ảnh đó biểu đạt đức hy sinh to lớn của tập thể nhiều nhà khoa học khắp nơi. Chúng là kết tinh công sức và trí tuệ của hàng triệu giờ lao động của hơn 200 nhà khoa học đến từ nhiều quốc gia trên thế giới, trong đó có cả sự tài trợ của trường Đại học Quốc gia Thành phố Hồ Chí Minh cho đài thiên văn East 
Asian Observatory, Hawaii, Hoa Kỳ. Chúng là biểu tượng của một ý niệm mỹ cảm khoa học tuyệt vời.

Bức ảnh được chụp lại bởi hệ thống kính viễn vọng Event Horizon Telescope (EHT)—Hệ thống 8 kính viễn vọng vô tuyến được đặt tại 5 châu lục khác nhau-quan sát và chụp lại trong một tuần vào tháng 4 năm 2017. Về mặt lý thuyết, hố đen vũ trụ là một vật thể vô hình khi đến ánh sáng cũng không thể thoát ra khỏi chân trời sự kiện (event horizon).

Tuy nhiên, các ý tưởng về việc quan sát gián tiếp hố đen thông qua bóng của nó trên đĩa bồi tụ (accretion disk) xung quanh đã được các nhà khoa học nghĩ đến, và được chứng minh là khả thi vào năm 1999 [3].

Nhưng cũng mãi đến năm 2006 thì những nỗ lực đầu tiên mới được nhà vật lý thiên văn Shep Doeleman (Harvard University, Mỹ) và các cộng sự tiến hành [4]. Và cũng mất đến 13 năm để chúng ta được chiêm ngưỡng thành quả đầu tiên.

Siêu hố đen lọt vào ống kính của Trái Đất nằm ở trung tâm thiên hà Messier 87 (M87), cách xa chúng ta khoảng 53.5 triệu năm ánh sáng và có trọng lượng lớn gấp 6.5 tỉ lần mặt trời, tức rộng khoảng 38 tỉ km. Tuy nhiên, từ góc nhìn của Trái Đất, hố đen này lại quá nhỏ để chúng ta có thể quan sát rõ ràng: tương đương với quan sát một quả bóng bàn trên mặt trăng (các bạn có thể xem từ phút 2:38 - 3:13 trong First Image of a Black Hole! để có thể hiểu rõ hơn về mặt tính toán [5]). Với phương pháp truyền thống, các nhà khoa học cần có 1 kính viễn vọng có kích cõ̃ bằng Trái Đất để quan sát.

Giải quyết cho vấn đề này, mạng lưới 8 kính thiên văn trong hệ thống EHT đã kết nối và tạo thành 1 ống kính viễn vọng đủ mạnh để quan sát quan sát hố đen tại thiên hà Sagittarius $\mathrm{A}^{*}$ của chúng ta, thiên hà $\mathrm{M} 87$, và các hạt nhân thiên hà hoạt động khác (Active Galactic Nucleus - AGN).

Dữ liệu thô để tạo nên bức ảnh lịch sử tương đương với các files mp3 dài 5000 năm, được lưu trữ trong khoảng nửa tấn ổ cứng và vận chuyển bằng phương thức vận tải thông thường đến Max Planck Institute for Radio Astronomy tại Bonn, Đức và Massachusetts Institute of Technology's Haystack Observatory tại Westford, Mỹ. Tại đây, đội ngũ các nhà nghiên cứu đã cùng hợp lực để xử lí khối dữ liệu khổng lồ này với thuật toán tái cấu trúc hình ảnh mới được phát triển.

Thực tế, trong năm 2016, phương pháp xử lí hình ảnh đã được nhóm nghiên cứu giới thiệu trên arXiv [6] và hội nghị IEEE Conference on Computer Vision and Pattern Recognition [7]. Đánh giá chính thống hơn được công bố vào ngày 27 tháng 10 năm 2016 với tựa đề Observing-and Imaging-Active Galactic Nuclei with the Event Horizon Telescope, nghiên cứu được đăng trên tạp chí Galaxies [ISSN: 2075-4434; ISI Web of Science ESCI; Scopus] thuộc nhà xuất bản Thụy Sĩ MDPI [8]. Trước đó, bài viết cũng đã được đăng trên hệ thống Preprints (cũng thuộc MDPI) vào ngày 12-6-2016 (DOI: 10.20944/preprints201607.0023.v1). Quả là một vinh dự khi một tạp chí mới chỉ nằm trong danh mục ESCI được các nhà nghiên cứu của một dự án quan trọng bậc nhất lịch sử khoa học lựa chọn đăng bài. 


\section{Article Metrics}

\section{Article access statistics}

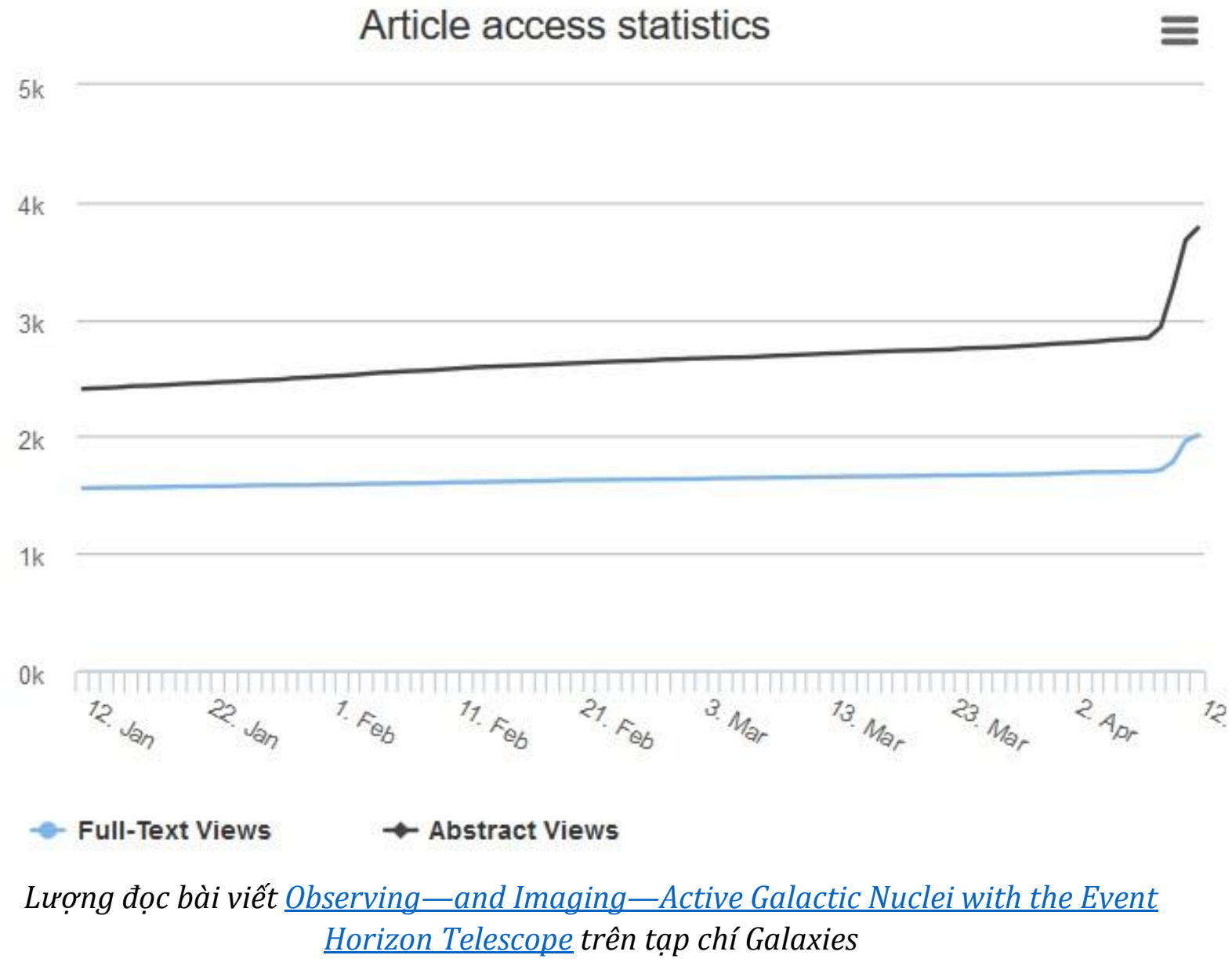

Tuy nhiên, lượng đọc bài nghiên cứu trên tạp chí Galaxies cũng rất nhỏ giọt, với chỉ dừng xấp xỉ 4000 lượt sau 3 năm. Ngay sau khi sự kiện bức ảnh hố đen được công bố, lượng tương tác đang có chiều hướng tăng rất nhanh.

Tiến sĩ Katie Bouman tại MIT đã có những đóng góp lớn trong phát triển thuật toán này [9]. Làm rõ hơn một chút về vấn đề này, đồng nghiệp của Bouman là Andrew Chael, trên Twitter cá nhân của mình, cho biết có đến 3 thư viện phần mềm độc lập được sử dụng trong ngiên cứu này.

Các thành viên trong nhóm đều đóng góp trong quá trình viết chương trình máy tính, xử lý lỗi và áp dụng chương trình vào thực tế tính toán dữ liệu từ EHT. Với cá nhân Katie Bouman, cô đã phát triển một khung tạo ảnh (imaging framework) để kiểm tra cả 3 bộ code kia. Một thư viện phần mềm khá đầy đủ được Chael đăng tải trên GitHub (https://github.com/achael/eht-imaging).

Cuối cùng, sau khi thành quả cuối cùng được chính thức công bố vào ngày 10 tháng 4 năm 2019, một chùm 6 nghiên cứu đã được công bố trên tạp chí Astrophysical Journal Letters [2017 JIF = 6.634; CiteScore = 5.07] [10-15]. Bức ảnh hố đen ở trung tâm thiên hà M87 và các nghiên cứu đã đưa ra bằng chứng thực tế của hố đen, vốn trước đó chỉ được chứng minh bằng tính toán. Đồng thời thuyết tương đối tổng quát mà Albert Einstein đưa 
ra hơn 100 năm trước cũng được khẳng định lại. Sau thành công bước đầu này, các nhà khoa học cũng sẽ tìm hiểu thêm về đĩa bồi tụ và dải tia chuẩn trực của hố đen. Sắp tới, các nhà khoa học cũng sẽ có thể công bố hình ảnh của hố đen tại trung tâm thiên hà Sagittarius $\mathrm{A}^{*}$ của chúng ta, vốn nhỏ, và ít hoạt động hơn so với hố đen tại M87. Khoa học, dù khô khan, nhưng lại rất đẹp vì những nỗ lực không ngừng nghỉ để thử thách giới hạn của tri thức.

Chúng ta hãy cùng tận hưởng một thành tựu lịch sử, được thai nghén trong hơn 20 năm, và cuối cùng thành công nhờ công sức hàng triệu giờ lao động và trí tuệ của 200 con người, và kèm theo đó là một chút băn khoăn kỳ lạ như cô Heather Schewedel:

— "Thế quái nào mà cái hình bánh donut này lại lên trang nhất được nhỉ?"

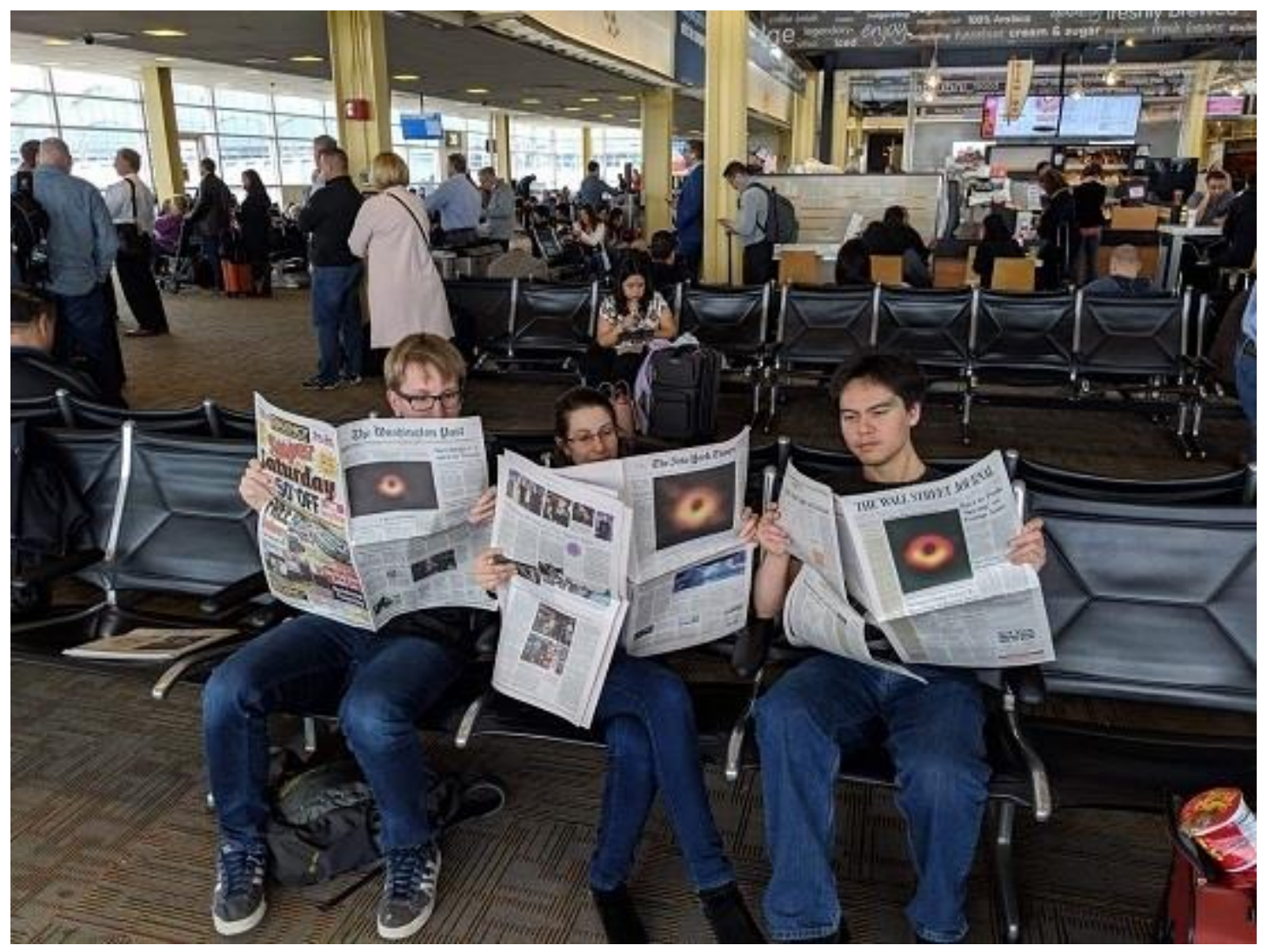

Thế mà chúng tôi cho cái ảnh này lên trang nhất đấy (Nguồn: Andrew Chael Twitter)

Là hỏi vậy, chứ ai cũng biết chỗ của bức hình là ở trang nhất không phải của một mà vô số các tờ báo uy tín nhất hành tinh, bằng cả dăm chục ngôn ngữ khác nhau. Chúng cũng lên tiêu điểm những ấn phẩm hàn lâm và truyền thông danh giá nhất: Science, Nature, The Guardian, The Verge, v.v.. và v.v.. [16-20]. 


\section{MDPI@MDPIOpenAccess·Apr 12}

MDPI Paper from Katie Bouman in @Galaxies_MDPI "Observing_and Imaging_Active Galactic Nuclei with the Event Horizon Telescope"

\section{Free to read at mdpi.com/2075-4434/4/4/... \#BlackHole}

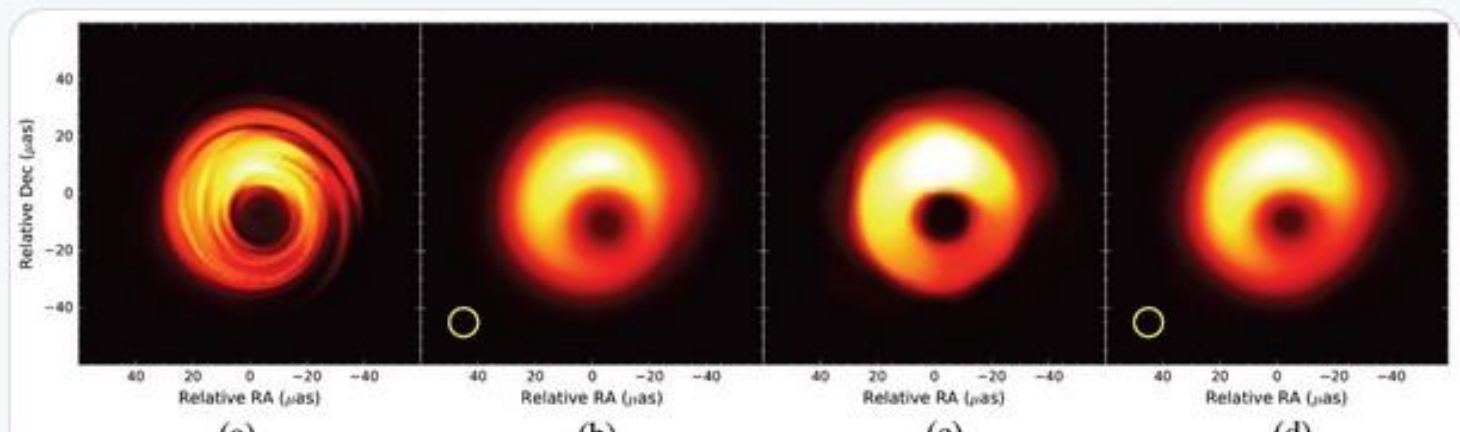

(a)

(b)

(c)

(d)

MIT Haystack Observatory

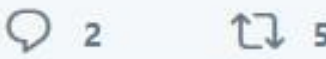

5

19

Còn nhà xuất bản MDPI thì cũng không quên bỏ lên một tweet, lặng lẽ và tinh tế nhắc lại bài đã đăng ngay khi sự kiện long trời lở đất diễn ra. Họ biết, giờ đây nhiều tạp chí khoa học hàng đầu có phần tị với xuất bản này của Galaxies (ISSN: 2075-4434). Với bước đà do nghiên cứu vang dội này mang lại, chẳng bao lâu nữa Galaxies sẽ được chỉ mục hóa vào danh mục SCIE, thậm chí SCI. Đây là sự trợ lực quan trọng cho MDPI, vốn đang gấp rút trên đường đua xuất bản khoa học giữa thời đại Plan $S$ chất chứa đầy những thay đổi mạnh mẽ hiện nay.

Notes: Published in SSHPA (13-04-2019): https://sc.sshpa.com/post/5449.

\section{References:}

[1] Schewedel, H. (April 10, 2019). We need to admit that the black hole photo isn't very good. Slate. Retrieved from https://slate.com/technology/2019/04/black-holephoto-just-isnt-very-good.html (April 16, 2019).

[2] Vuong, Q. H. (2018). The (ir) rational consideration of the cost of science in transition economies. Nature Human Behaviour, 2(1), 5, DOI: 10.1038/s41562-017-0281-4.

[3] Falcke, H., Melia, F., \& Agol, E. (1999). Viewing the shadow of the black hole at the galactic center. The Astrophysical Journal Letters, 528(1), L13.

[4] Nadis, S. (April 10, 2019). Why the Event Horizon Telescope took so long to image a black hole. Astronomy. Retrieved from https://astronomy.com/news/2019/04/the-road-to-imaging-a-black-hole (April 16, 2019). 
[5] Veritasium. (April 10, 2019). First image of a black hole! [Video file]. Retrieved from https://www.youtube.com/watch?v=S GVbuddri8\&t.

[6] Johnson, M. D. (2016). Stochastic optics: a scattering mitigation framework for radio interferometric imaging. arXiv preprint arXiv:1610.05326, DOI: $10.3847 / 1538$ $\underline{4357 / 833 / 1 / 74}$.

[7] Bouman, K. L., Johnson, M. D., Zoran, D., Fish, V. L., Doeleman, S. S., \& Freeman, W. T. (2016). Computational imaging for VLBI image reconstruction, in Proceedings of the IEEE Conference on Computer Vision and Pattern Recognition, Las Vegas, 2016 (pp. 913-922).

[8] Fish, V., Akiyama, K., Bouman, K., Chael, A., Johnson, M., Doeleman, S., ... \& Event Horizon Telescope Collaboration. (2016). Observing-and imaging-active galactic nuclei with the Event Horizon Telescope. Galaxies, 4(4), 54, DOI: $10.3390 /$ galaxies 4040054 .

[9] Ellis-Petersen, H. (April 11, 2019). Katie Bouman: the 29-year-old whose work led to first black hole photo. The Guardian. Retrieved from https://www.theguardian.com/science/2019/apr/11/katie-bouman-black-holephoto (April 11, 2019).

[10] The Event Horizon Telescope Collaboration. (2019). First M87 Event Horizon Telescope results. I. The shadow of the supermassive black hole. Astrophysical Journal Letters, 875(1), L1, DOI: 10.3847/2041-8213/ab0ec7.

[11] The Event Horizon Telescope Collaboration. (2019). First M87 Event Horizon Telescope results. II. Array and instrumentation. Astrophysical Journal Letters, 875(1), L2, DOI: 10.3847/2041-8213/ab0c96.

[12] The Event Horizon Telescope Collaboration. (2019). First M87 Event Horizon Telescope results. III. Data processing and calibration. Astrophysical Journal Letters, 875(1), L3, DOI: 10.3847/2041-8213/ab0c57.

[13] The Event Horizon Telescope Collaboration. (2019). First M87 Event Horizon Telescope results. IV. Imaging the central supermassive black hole. Astrophysical Journal Letters, 875(1), L4, DOI: 10.3847/2041-8213/ab0e85.

[14] The Event Horizon Telescope Collaboration. (2019). First M87 Event Horizon Telescope results. V. Physical origin of the asymmetric ring. Astrophysical Journal Letters, 875(1), L5, DOI: 10.3847/2041-8213/ab0f43.

[15] The Event Horizon Telescope Collaboration. (2019). First M87 Event Horizon Telescope results. VI. The shadow and mass of the central black hole. Astrophysical Journal Letters, 875(1), L6, DOI: 10.3847/2041-8213/ab1141.

[16] Castelvecchi, D. (2019). Black hole pictured for first time - in spectacular detail. Nature, DOI: d41586-019-01155-0.

[17] Clery, D. (2019). For the first time, you can see what a black hole looks like. Science, DOI: $10.1126 /$ science.aax6474. 
[18] Editorial. (April 12, 2019). The Guardian view on the first picture of a black hole: seeing is believing. The Guardian. Retrieved from https:/www.theguardian.com/commentisfree/2019/apr/12/the-guardianview-on-the-first-picture-of-a-black-hole-seeing-is-believing (April 16, 2019).

[19] Griggs, M. B. (April 10, 2019). See the first image ever taken of a supermassive black hole. The Verge. Retrieved from https://www.theverge.com/2019/4/10/18303661/first-picture-black-holesagittarius-event-horizon-telescope (April 16, 2019).

[20] Koren, M. (April 10, 2019). An extraordinary image of the black hole at a galaxy's heart. The Atlantic. Retrieved from https://www.theatlantic.com/science/archive/2019/04/black-hole-eventhorizon-telescope/586846/ (April 16, 2019). 